\title{
Calculation of the required noise reduction of a engine exhaust system
}

\author{
Olga $\mathrm{M}$ atasova*, Vladimir Tupov \\ B auman M oscow State Technical University, 105005, M oscow, Russia
}

\begin{abstract}
The problem of reduction of internal combustion engine (ICE) noise is most urgent at present when the fleet of vehicles has significantly expanded and their power has increased. R eactive mufflers are of ten used to solve it. The initial stage in the design of mufflers is a theoretical assessment of the frequency spectrum of sound power level (SPL) of an unmuffled ICE noise source, comparing it with the calculated maximum permissible spectrum and determining the required amount of its reduction in standard frequency bands. The paper presents a method for calculating the required noise reduction of a transport engine exhaust using the example of a twostroke ICE of the class of $125 \mathrm{~cm}^{3}$. Thus, the designer has the opportunity to develop mufflers, the acoustic efficiency of which meets the calculated required noise reduction values, already at an early stage of the vehicle design.
\end{abstract}

\section{Introduction}

Currently, due to technological progress, the number of noise sources is constantly growing. The problem of noise control is becoming more and more urgent since exceeding the normative values of noise exposure on a person adversely affects his health.

One of the most common noise sources in the urban environment is transportation with which people regularly interact. $M$ otor vehicles equipped with two-stroke carburetor internal combustion engines (ICE) with a crank-chamber purge system have especially high noise levels.

The most powerful noise source in a transport engine is its exhaust system, so the development of high-performance mufflers is of paramount importance. Taking into account the fact that the muffler affects the process of filling the working cylinder with a gas-air mixture and thereby affects the power indicators of the engine, its development should be carried out even at the early stages of ICE design. A the same time, there is no possibility of an experimental assessment of the sound power of unmuffled emission noise and its comparison with the permissible values. Hence, it becomes necessary to develop and apply calculation methods for determining the frequency spectrum of sound power level (SPL) of the ICE exhaust noise, to develop a method for calculating the maximum permissible SPL spectrum of the exhaust noise, the comparison of which makes it possible to find the required noise reduction of a given source depending on the sound frequency presented in the works.

\footnotetext{
${ }^{*}$ Corresponding author: olgasanm@ rambler.ru
} 
[1-10]. The calculation in octave frequency bands of these parameters, which are the initial data for the design of mufflers, is an important step in the creation of highly effective means of noise reduction of engine exhaust. This paper is devoted to the solution of this problem by the example of calculating the required noise reduction of the exhaust of a two-stroke carburetor engine of the class of $125 \mathrm{~cm}^{3}$.

\section{Formulation of the research problem}

The question of the need to use noise suppression means is resolved by comparing the actual corrected sound power level of the engine noise with the permissible level recalculated in SPL. If this value is exceeded, individual noise sources, for example, the exhaust, should be normalized according to the maximum permissible spectrum, determining the frequency bands in which it is necessary to reduce its levels. The amount of required SPL reduction of the exhaust noise in each frequency band is calculated by the formula:

$$
\Delta L_{r e q_{v}}=L_{W_{v}}-L_{W_{p^{2} r_{v}}}, \mathrm{~dB},
$$

where $L_{W_{v}}$ - is the SPL of unmuffled exhaust noise; $L_{W_{p e r}}$ - is the maximum permissible SPL of this noise source.

Thus, to determine the value of the required noise reduction of the exhaust, for example, in the octave frequency bands, it is necessary, first of all, to calculate the SPL spectrum of the unmuffled noise of the exhaust noise and its maximum permissible spectrum.

\section{Research of the process of noise production at the ICE exhaust}

The exhaust process begins when the piston moves downward at the moment the piston opens the exhaust port (Fig.1). A typical curve of the volumetric velocity of exhaust gases flowing out through the exhaust port for a short exhaust line without a muffler is shown in fig. 2. At the initial moment, the pressure inside the cylinder reaches significant values; therefore, the volumetric velocity of the outflowing gases first increases rapidly as the exhaust ports open, and the linear velocity of the gases reaches the sound speed. How ever, as the exhaust gases flow out, the pressure difference betw een the pressure inside the cylinder and the outside decreases, as a result of which the volumetric velocity of the outgoing gases also begins to decrease. With the further downward movement of the piston, the purge ports open, and purge air from the sub-piston cavity begins to flow into the cylinder, where the pressure at this time is somewhat greater than the pressure in the cylinder. The process of exhaust gases flowing out of the cylinder until the purge gases enter the cylinder is called free exhaust. On curve 1 of Figure 2, this moment is marked with the exact G. Further, the purge gases, filling the cylinder, displace the exhaust gases remaining in it after free exhaust, therefore the second stage of the exhaust process is called purge-filling. In the figure, this is the GF section. A slight increase in the volumetric velocity of the outflowing gases in this section is due to a slight increase in pressure in the cylinder due to the entry of purge gases into it. Finally, the last stage of the exhaust is the FL section, when the piston moves up, the purge ports are closed, and the exhaust port is still open. This site is called an additional exhaust.

The exhaust process begins when the piston moves downward at the moment the piston opens the exhaust port (Fig. 1). 


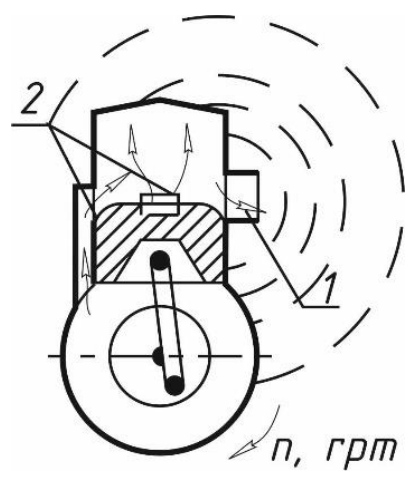

Fig. 1. Diagram of the exhaust process: 1 - exhaust port, 2 - purge ports [1].

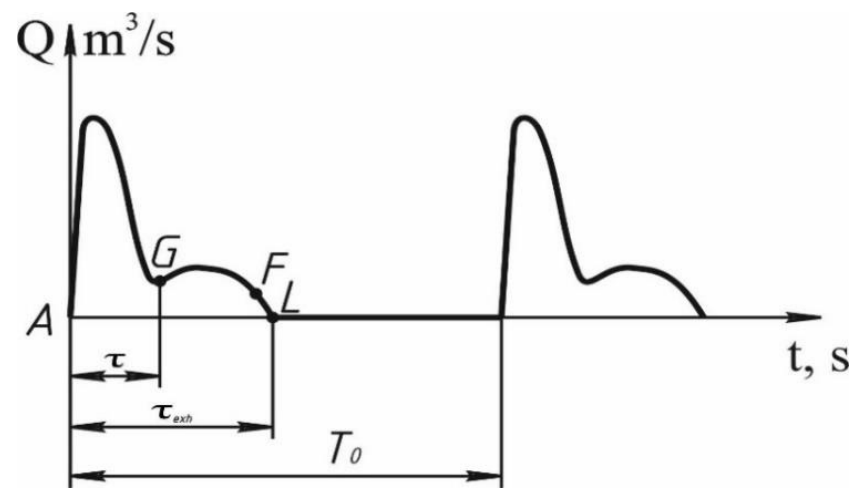

Fig. 2. Dependence of the volumetric velocity of outgoing gases on time [1].

The gases reach their highest velocity during the free exhaust period, when the hottest gases, whose energy reaches $85 \%$ of the total energy of the exhaust gases, are exhausted. Therefore, when calculating, we will consider that the intensive process of noise generation occurs only in the free exhaust area, and the processes of blowing - filling and additional exhaust will not be taken into account $[1,4]$.

\section{Determination of constant parameters for calculating SPL noise of exhaust}

The following parameters are the permanent ones that characterize the emission process in terms of noise generation [1]:

- main process frequency $f_{0}(\mathrm{~Hz})$ and the period of repetition of the disturbing impulses $\mathrm{T}_{0}=1 / f_{0}(s)$;

- time $\tau$, during which there is a free flow (s);

- $\quad$ the volume of gases $V$, flowing out during the period of the free exhaust $\left(m^{3}\right)$;

- constants of the idealized process $K\left(\mathrm{~m}^{3} \mathrm{rad} / \mathrm{s}\right)$ and $\beta(\mathrm{rad} / \mathrm{s})$.

K nowing the initial parameters of the engine considered for the example: $n=5000 \mathrm{rpm}-$ is the number of revolutions of the engine crankshaft and $\varphi_{\text {exh }}=150^{\circ}$ - is the phase of the exhaust process, we determine the above values.

Since exhaust occurs with every revolution of the engine crankshaft, the fundamental frequency of the process is:

$$
f_{0}=n / 60, H z \text {. }
$$


here $f_{0}=83,3 \mathrm{~Hz}$.

The repetition period of the process is calculated by the formula:

$$
\mathrm{T}_{0}=1 / f_{0}, \mathrm{~s},
$$

and is for a given engine $\mathrm{T}_{0}=12 \cdot 10^{-3} \mathrm{~s}$.

The duration of the noise generation process $\tau$ is determined using the following assumptions [1]:

- the end of the free exhaust process is the moment when the piston passes the bottom dead center;

- the duration of the process of the most intense noise generation is equal to half the duration of the entire exhaust process.

Thus, the exhaust time and, as a consequence, the noise generation time, are calculated using the following formulas:

$$
\begin{aligned}
& \tau_{\text {exh }}=\mathrm{T}_{0} \varphi_{\text {exh }} / 360, \mathrm{~s} ; \\
& \tau=0,5 \tau_{\text {exh }}, \mathrm{s} .
\end{aligned}
$$

As the volume $V$ corresponding to a given time $\tau=2,5 \cdot 10^{-3} \mathrm{~s}$, one can take the volume obtained as a result of the adiabatic outflow of the initial volume equal to the volume of the cylinder at the moment of exhaust start $V_{\mathrm{B}}=2 \cdot 10^{-4} \mathrm{~m}^{3}$. The parameters of the gas in the cylinder at the start of the exhaust are taken as the initial parameters during the outflow, and the atmospheric parameters are taken as the final parameters.

Next, the constant $K$ of the ideal ized process is cal culated:

and constant $\beta$ :

$$
\kappa=2 \pi V / \tau
$$

$$
\beta=\pi / \tau \text {. }
$$

The values of these constants for the engine under consideration are $K=0,503 \mathrm{~m}^{3} / \mathrm{s}$ and $\beta=1,257 \cdot 10^{3} \mathrm{rad} / \mathrm{s}$.

The calculation of SPL of the noise source is divided into two parts [1]:

1. Determination of spectrum components in the frequency range $\omega_{i}<\beta \sqrt{2}$;

2. Determination of spectrum components in the frequency range $\omega_{i}>\beta \sqrt{2}$.

Conventionally, the first range can be called the low-frequency part of the spectrum, and the second range - the high-frequency part of the spectrum.

The limit frequency separating the low-frequency and high-frequency ranges are determined by the formula:

$$
\omega_{\text {lim }}=\beta \sqrt{2}, \mathrm{rad} / \mathrm{s}
$$

$\omega_{\text {lim }}=1,778 \mathrm{rad} / \mathrm{s}$.

A ccordingly, the number of the harmonic, starting from which it is possible to switch to the formulas of the high-frequency range, will be:

$$
i_{\text {lim }}=\omega_{\text {lim }} /\left(2 \pi f_{0}\right) \text {. }
$$

The resulting value is rounded up to a whole number. When substituting the numerical values of the previously calculated values, we obtain that up to the 4th harmonic we use the formulas for low frequencies given below.

\section{Calculation of the low-frequency spectrum of sound power levels of unmuffled outlet noise}

For low frequencies, the SPL is calculated using the formula: 


$$
L_{W_{i}}=98+20 \lg \left(i f_{0}\right)+20 \lg Q_{m i}, d B,
$$

where $i$ is the ordinal number of the harmonic component of the SPL spectrum; $Q_{m i}$ is the amplitude of the volumetric vibrational velocity of the exhaust gases at the outlet of the engine exhaust system, $\mathrm{m}^{3} / \mathrm{s}$.

The value $Q_{m i}$ is preliminarily determined from the expression:

$$
Q_{m i}=\left(2 K / T_{0}\right) \beta\left(4 \beta^{4}+\omega_{i}^{4}\right)^{-0,5}, m^{3} / s,
$$

where $\omega_{\mathrm{i}}=2 \pi i f_{0}, \mathrm{rad} / \mathrm{sis}$ the circular frequency of the $i$-th harmonic of the spectrum.

Calculations for the first four harmonics were performed in $\mathrm{M}$ athcad15 using formulas (10) and (11), and their results are presented in Table. 1.

Table 1. Calculation results of low-frequency harmonics of the SPL spectrum.

\begin{tabular}{|c|c|c|c|c|}
\hline $\mathrm{i}$ & $f_{i}$ & $L_{W_{i}}$ & $f_{a v_{v}}$ & $L_{W_{v}}$ \\
\hline & $\mathrm{Hz}$ & $\mathrm{dB}$ & $\mathrm{Hz}$ & $\mathrm{dB}$ \\
\hline 1 & 83,3 & 106,8 & 63 & 106,8 \\
\hline 2 & 166,6 & 112,4 & 125 & 112,4 \\
\hline 3 & 249,9 & 114,4 & 250 & 117,4 \\
\cline { 1 - 2 } 4 & 333,2 & 114,3 & & \\
\hline
\end{tabular}

Next, the octave sound power levels are determined. The first two-octave bands with average frequencies $f_{\text {av }}=63 \mathrm{~Hz}$ and $f_{a v}=125 \mathrm{~Hz}$ each contain one harmonic ( $i=$ 1 and $i=2$ ), each of which determines the total SPL in the corresponding octave. The thirdoctave band with a geometric mean frequency $f_{a v_{v}}=250 \mathrm{~Hz}$ contains two harmonic components $i=3$ and $i=4$. Adding them according to the nomogram (Fig. 3), the total level in the given octave frequency band $L_{\text {Woct }}=117,4 \mathrm{~dB}$ is calculated.

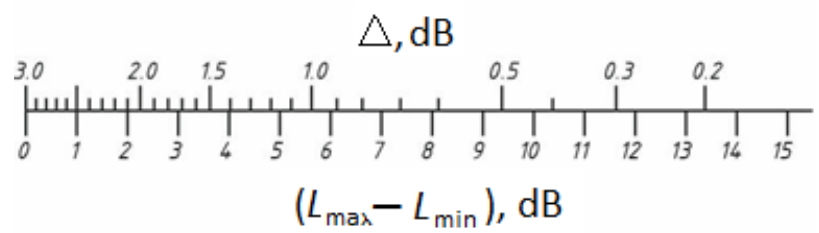

Fig. 3. Nomogram for adding levels [1].

\section{Calculation of the high-frequency spectrum of the sound power levels of the unduffleded outlet noise}

The sound power level of exhaust noise in octave bands at high frequencies is calculated [1] by the formula:

$$
L_{W_{v}}=96+20 \lg V-40 \lg \tau+10 \lg \left(f_{0} / f_{a v_{v}}\right), d B .
$$

For the frequency band with $f_{a v}=500 \mathrm{~Hz}$ this value is $118,3 \mathrm{~dB}$. For each of the subsequent octave bands, the sound power decreases by $3 \mathrm{~dB}$ as the mid frequencies of the octaves are doubled.

The cal culation according to the formula (12) executed in M athcad15, and the calculation results are given in the table. 2 . 
Table 2. The results of calculating the high-frequency harmonics of the SPL spectrum.

\begin{tabular}{|c|c|}
\hline$f_{a v_{v}}$ & $L_{W_{v}}$ \\
\hline $\mathrm{Hz}$ & $\mathrm{dB}$ \\
\hline 500 & 118,3 \\
1000 & 115,3 \\
2000 & 112,3 \\
4000 & 109,3 \\
8000 & 106,3 \\
\hline
\end{tabular}

The resulting octave spectrum of the sound power level of the exhaust process is shown in fig. 4.

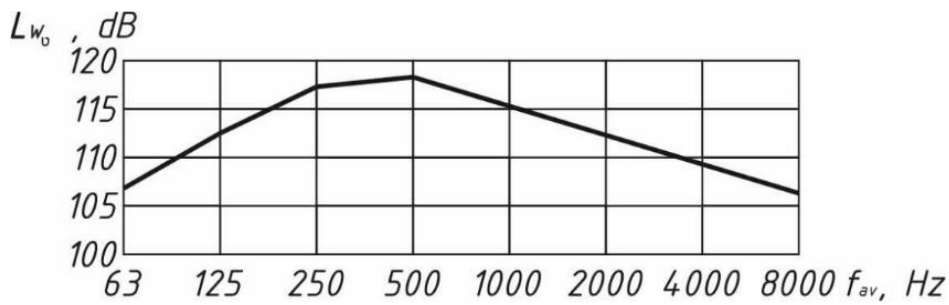

Fig. 4. Octave spectrum of unmuffled exhaust noise of the investigated ICE.

\section{Determination of the permissible SPL spectrum of the ICE exhaust noise}

The permissible noise levels of motorcycle engines and their main sources can be obtained using the standard values for the noise of motor vehicles specified in GOST R 41.41-2001 [2]. Engine noise is 85 ... 94\% of all motorcycle noise. W ith a certain margin in terms of the noise level of the designed motor vehicles, it can be assumed that $80 \%$ of the sound energy of the motorcycle is emitted by the engine [3]. A llowable corrected SPL of the engine in the vehicle test mode:

$$
L_{W_{\mathrm{A}_{p e r}}}=L_{\mathrm{A}_{\text {per }}}+20 \lg \frac{r}{r_{0}}-10 \lg \frac{\Phi}{2 \pi}+10 \lg \left(\frac{\rho_{a_{0} a_{0}}}{\rho_{a} c_{a}}\right)+\frac{\beta_{\mathrm{a}} r}{1000}-0,7, d B \mathrm{~A},
$$

where $\rho_{a}, c_{a}$ and $\rho_{a_{0}}, c_{a_{0}}$ is the air density and sound speed in it, respectively, at the time of measurement and under normal atmospheric conditions (when the air temperature changes from -5 to $\left.+30{ }^{\circ} \mathrm{C}\left|10 \lg \frac{\rho_{a_{0} c_{0}}}{\rho_{a} c_{a}}\right| \leq 0,2 d B A\right) ; \beta_{a}$ is the sound attenuation coefficient in the atmosphere, $\mathrm{dBA} / \mathrm{km}$ (in this case $\frac{\beta_{a} \cdot r}{1000} \leq 0,3 \mathrm{dBA}$ ); $L_{\mathrm{A}_{\text {per }}}$ is the maximum permissible sound level $(\mathrm{dB} A)$ of a motor vehicle with engine displacement from $80 \mathrm{~cm}^{3}$ and up to 175 $\mathrm{sm}^{3}$ inclusive following GOST R 41.41 - 2001 [2]; $r$ - is the distance from the acoustic center of the motor vehicle to the measuring microphone at the time of recording the sound level meter readings $(\mathrm{m})$; $\Phi$ - is the directivity factor of noise radiation in the direction of the microphone; $r_{0}=1 \mathrm{~m}$.

Formula (13) does not take into account the absorption of sound by the asphal t or concrete pavement of the measuring section of the road, which leads to some decrease in the value of $L_{W_{\text {Aper }}}$, but not more than 0,5 dB A [3]. This error can be tolerated since it causes a small margin for the noise level of the motor vehicle. 
If we neglect the fourth and fifth terms of the right-hand side of equation (13) due to their smallness and substitute the values $r \approx 7,5 \mathrm{~m}$ and $\Phi \approx 1$, then this formula will take the form:

$$
L_{W_{\text {Aper }}}=L_{\mathrm{A}_{\text {per }}}+24,8, d B \mathrm{~A} \text {. }
$$

Since the exhaust noise is set in the form of a frequency of SPL spectrum, it is convenient to use the permissible spectrum for normalization, the values of which are in each $i$-th standard frequency band (for example, octave) can be determined by the formula [3]:

$$
L_{W_{\text {perv }}}=L_{W_{A_{p e r}}}-10 \lg \left(m_{b} n_{n s}\right)+\Delta_{n s}-\Delta_{A_{v}}, d B,
$$

where $v=1,2,3, \ldots m_{b} ; m_{b}$ - is the number of frequency bands in which the SPL exceeds the permissible, calculated by the formula (15); $n_{n s}$ - is the number of noise sources taken into account (usually exhaust, intake, ICE housing); $\Delta_{n s}$ - is the correction set for each noise source taking into account the technical complexity and unit cost of its muffling; $\Delta_{\mathrm{A}_{v}}$ is the correction for the shape of the frequency response "A " of the sound level meter (see Table 3).

The technical difficulties of muffling depend on some reasons, which are generally difficult to quantify, therefore it is not possible to accurately determine the correction $\Delta_{n s}$. For exhaust noise of a motorcycle engine, its value can be taken approximately: $\Delta_{n s}=-3$ [3].

The calculation of the extremely permissible spectrum of exhaust noise is carried out in octave frequency bands. Since the real number of octave bands in which there is an excess of the maximum permissible spectrum is unknown in advance, we assume that it is present in all bands, i.e., we take $m_{b}=8$.

Substituting the values of its terms given above into the right side of equation (15), we obtain the extremely permissible SPL spectrum of exhaust noise from a motorcycle engine of the class of $125 \mathrm{~cm}^{3}$. The cal culation results calculated in M athcad15 are shown in table 3 and graphically shown in fig. 5.

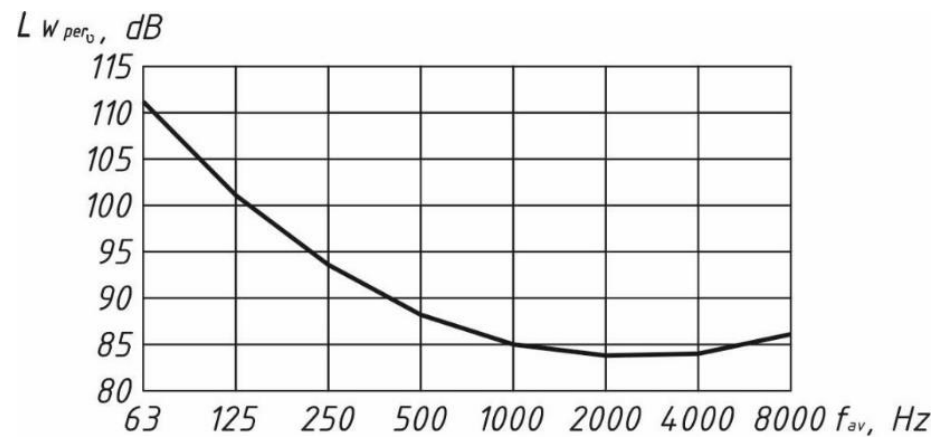

Fig. 5. Allowable octave SPL spectrum of exhaust noise of motorcycle ICE of $125 \mathrm{~cm}^{3} \mathrm{class}$.

Table 3. Extremely permissible octave SPL spectrum of exhaust noise from motorcycle ICE and frequency correction "A" of the sound level meter.

\begin{tabular}{|c|c|c|c|c|c|c|c|c|}
\hline$f_{a v_{v}}, H z$ & 63 & 125 & 250 & 500 & 1000 & 2000 & 4000 & 8000 \\
\hline$\Delta_{\mathrm{A}_{v}}, d B$ & $-26,2$ & $-16,1$ & $-8,6$ & $-3,2$ & 0 & $+1,2$ & $+1,0$ & $-1,1$ \\
\hline$L_{\text {Wper }_{v}}, d B$ & 111,2 & 101,1 & 93,6 & 88,2 & 85,0 & 83,8 & 84,0 & 86,1 \\
\hline
\end{tabular}




\section{Calculation of the amount of the required SPL reduction of the ICE exhaust noise in octave bands}

K nowing the sound power level of exhaust noise without muffler of motorcycle ICE of the class of $125 \mathrm{~cm}^{3}$ in octave frequency bands and the corresponding extremely permissible octave spectrum, let us calculate the required reduction in SPL of exhaust noise in octaves. The calculation is carried out according to the formula (1) using the above values. The results of the calculation performed in M athcad15 are presented in table 4 and shown graphically in fig.6.

Table 4. Required SPL reduction of exhaust noise from a motor engine of the class of 125 $\mathrm{cm}^{3}$ in octaves.

\begin{tabular}{|c|c|c|c|c|c|c|c|c|}
\hline$f_{a v_{v}}, H z$ & 63 & 125 & 250 & 500 & 1000 & 2000 & 4000 & 8000 \\
\hline$\Delta L_{W_{\text {requ }}}, d B$ & 0 & 11,3 & 23,8 & 30,1 & 30,3 & 28,5 & 25,3 & 20,2 \\
\hline
\end{tabular}

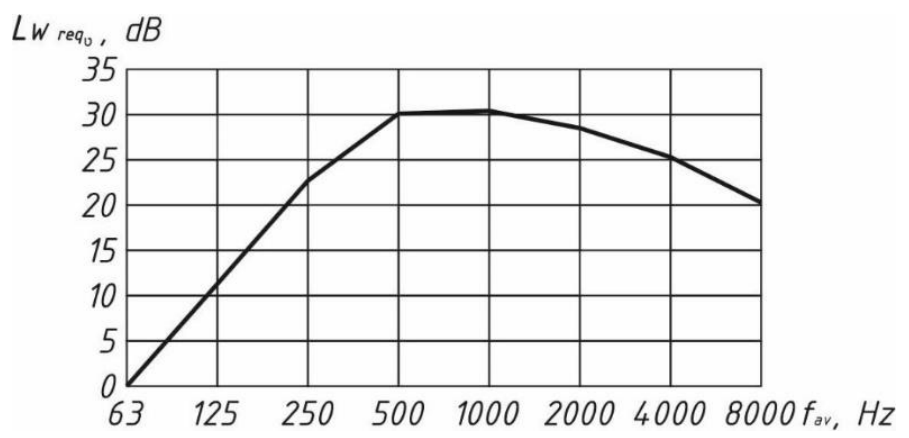

Fig. 6. Required SPL reduction of exhaust noise from a motor engine of the class of $125 \mathrm{~cm}^{3}$ in octaves.

Thus, the exhaust noise of ICE of a motorcycle of the class of $125 \mathrm{~cm}^{3}$ must be reduced practically in the entire frequency range, except for the octave band with $f_{a v}=63 \mathrm{~Hz}$. The highest values of required noise reduction of the exhaust are observed at mid and high frequencies, reaching $30 \mathrm{~dB}$ in octave bands with $f_{a v}=500$ and $1000 \mathrm{~Hz}$.

\section{Conclusion}

This paper presents a method for the theoretical estimation of the SPL of the ICE emission noise, as well as a method for determining the maximum permissible spectrum necessary for the implementation of emission noise regulation at the early stages of the vehicle design. Thus, it becomes possible to develop the design of the exhaust muffler, the acoustic efficiency of which corresponds to the calculated values of the required reduction in standard frequency bands. Thanks to this, the designer can create highly efficient mufflers at the same time as the vehicle is being developed. 


\section{References}

1. A.A. Strokin, Method for calculating the noise of pulsating sources. Methodical guidelines for diploma design, - M .: Bauman M oscow Higher Technical School, 3-40, (1975) [ in Russian]

2. GOST R (all-Union State Standard) 41.41-2001. Uniform provisions concerning the approval of motorcycles in relation to noise emissions. [ in Russian]

3. V.M. Kondrashov, Y u.S. Grigoriev, V.V. Tupov et al. Two-stroke carburetor internal combustion engines, - M.: ed. M echanical engineering, 215 - 270, (1990) [ in Russian]

4. E.Y a. Y udin, A.A. Strokin, , J. Higher Educ. Inst. M echanical engineering, 9, 86-90, (1972) [ in Russian]

5. V.V.Tupov, Reducing the noise of motorcycle engines, in proceedings of B auman M oscow Higher Technical School, 507, 86 - 110 (1988) [ in Russian]

6. J.W. Sullivan, Modeling of engine exhaust system noise, A erodynamic noise in technology: Translated from English. M .: M ir, 233 - 256 (1983

7. A.I. Komkin, N.A. Nikiforov, R.M. Simkin On the design of automobile exhaust systems, in Proceedings of the Sixth International congress on sound and vibration, Copenhagen, Denmark (1999)

8. A.I. K omkin, M.A. M ironov, A .I. Bykov, A coust. Phys. 63 (4), 385 (2017)

9. N. D.Chaynov, V. A.Markov, A. A. Savastenko, Structural Noise and Acoustic Characteristics Improvement of Transport Power Plants, in IOP Conference Series: $M$ aterials Science and Engineering, 327 (2) C. 022069 (2018)

10. R. I. Rakhmatov, V. E. K rutolapov, V. N. Zuzov, V ibroengin. Proc. 25, 135 (2019) 